\title{
Tratamento da insuficiência cardíaca terminal através da correção da insuficiência mitral secundária e remodelação ventricular
}

\author{
Enio BUFFOLO ${ }^{*, * *}$, Ivan Machado de PAULA ${ }^{*, * *}$, João Nelson R. BRANCO*,**, Antônio Carlos \\ C. CARVALHO ${ }^{*, * *}$, Cyrillo MANTOVANI ${ }^{*, * *}$, Guido CAPUTI ${ }^{*, * *}$, Luciano Figueiredo AGUIAR ${ }^{*, * *}$
}

RBCCV 44205-548

Buffolo E, Paula I M, Branco J N R, Carvalho A C C, Mantovani C, Caputi G, Aguiar L F - Tratamento da insuficiência cardíaca terminal através da correção da insuficiência mitral secundária e remodelação ventricular. Rev Bras Cir Cardiovasc 2001; 16(3): 203-11.

RESUMO: Introdução: A sobrevida de pacientes com miocardiopatia e insuficiência mitral secundária em classe funcional IV é muito pequena em curtos períodos de observação, apesar dos progressos consideráveis obtidos com o tratamento médico. Tem sido demonstrado que o aparecimento de insuficiência mitral secundária piora o prognóstico e a qualidade de vida e que a correção da insuficiência mitral permite melhor controle do paciente.

Objetivo: O presente trabalho propõe o implante de uma prótese em posição mitral que elimina a insuficiência mitral e remodela a base do ventrículo esquerdo e o seu eixo longitudinal.

Casuística e Métodos: Analisamos 33 pacientes com insuficiência cardíaca refratária sob terapêutica máxima operados entre dezembro/95 e setembro/99. O grupo etário variou de 25 a 78 anos (mediana 58) sendo $57,7 \%$ do sexo masculino. O período de observação variou de 4 meses a 4 anos (mediana 22 meses). Quanto à etiologia, em 15 pacientes era isquêmica, em 13 dilatada, em 3 chagásica, em 1 pós-parto e em 1 viral.

Resultados: Ocorreram 3 óbitos na fase hospitalar (3/33) e 2 na tardia (2/30), estando 28 pacientes em observação. Neste intervalo de seguimento, $88 \%$ dos pacientes melhoraram 1 ou 2 classes funcionais, a fração de ejeção subiu de 20 a $36 \%$, apesar da eliminação da fração regurgitante, o volume efetivo melhorou consideravelmente $(58$ para $80 \mathrm{ml}$ ) entre a aferição pré-operatória e a última evolução.

Conclusão: A insuficiência cardíaca refratária com insuficiência secundária moderada a severa pode sofrer efetiva paliação com a eliminação de regurgitação e remodelação do ventrículo esquerdo, implantandose prótese valvar no anel atrioventricular esquerdo.

DESCRITORES: Baixo débito cardíaco, cirurgia. Insuficiência da valva mitral, cirurgia. Remodelação ventricular. Implante de prótese de valva. Baixo débito cardíaco, complicações. Insuficiência da valva mitral, etiologia. Baixo débito cardíaco, análise de sobrevivência.

Trabalho realizado na Escola Paulista de Medicina e no Hospital do Coração da Associação do Sanatório Sírio. São Paulo, SP, Brasil. Apresentado ao $27^{\circ}$ Congresso Nacional de Cirurgia Cardíaca. Rio de Janeiro, RJ, 23 a 25 de março, 2000.

*Da Escola Paulista de Medicina.

** Do Hospital do Coração da Associação do Sanatório Sírio.

Endereço para correspondência: Enio Buffolo. Rua Borges Lagoa, 783 - 5a andar - Vila Clementino. São Paulo, SP, Brasil. CEP 04038-031 Tel.: (11)

5574-6611. Fax (11) 5573-0303. e-mail: ebuffolo@ zaz.com.br 
Buffolo E, Paula I M, Branco J N R, Carvalho A C C, Mantovani C, Caputi G, Aguiar L F - Tratamento da insuficiência cardíaca terminal através da correção da insuficiência mitral secundária e remodelação ventricular. Rev Bras Cir Cardiovasc 2001; 16(3): 203-11.

\section{INTRODUÇÃO}

O tratamento cirúrgico alternativo ao transplante cardíaco em casos de insuficiência cardíaca terminal constitui ainda um grande desafio a ser vencido.

A importância socioeconômica de pacientes nestas condições é muito grande. Em estatísticas norte-americanas estima-se que cerca de 400.000 novos casos são diagnosticados anualmente, representando 15 milhões de internações e 200.000 óbitos/ ano (1-3).

Estes números representam custos de 10 biIhões de dólares/ano, sendo $75 \%$ de custos hospitalares e $25 \%$ com cuidados ambulatoriais.

No Estado de São Paulo, dados coletados do SUS demonstram que os custos das internações por afecções cardiovasculares consomem cerca de $20 \%$ das verbas da Saúde.

Sabe-se que os pacientes portadores de insuficiência cardíaca em fases avançadas (classes III e IV), refratárias ao tratamento médico, beneficiamse extraordinariamente com o transplante cardíaco. Todavia, o reduzido número de doadores impede a possibilidade de estender esta terapêutica a um contingente maior de pacientes está muito longe de ser ideal.

Com isto, formas alternativas de tratamento cirúrgico têm despertado interesse. Dentre estas, já foram utilizadas a cardiomioplastia (4-7), ventriculectomia parcial (8-11), cirurgia de Jatene e Dor (12), correção da insuficiência mitral ${ }^{(13-16)} \mathrm{e}$, mais recentemente, a estimulação multisítio (17-20).

Os resultados a médio e a longo prazo destas técnicas constituem motivo de grande polêmica, estando sob julgamento e merecendo correções de rumo.

Resultados mais consistentes têm sido obtidos com a diminuição da insuficiência mitral secundária através da plastia valvar.

Os satisfatórios resultados iniciais são mantidos ao final de 4 anos de seguimento e são atribuídos à diminuição da fração regurgitante, sendo que o ventrículo esquerdo aproveita todo seu gasto energético numa sístole efetiva.

É reconhecido também o fato de que a insuficiência mitral quando aparece nas fases terminais onera consideravelmente o manejo clínico, a qualidade de vida e a sobrevivência.

$\mathrm{Na}$ insuficiência cardíaca terminal que é a via final comum de várias cardiomiopatias de discutidas etiologias, a insuficiência mitral aparece pela distorção da base dos músculos papilares, da esfericidade do ventrículo esquerdo e pela dilatação ostial (21).

Nestas situações a cavidade ventricular esquerda assume a forma arredondada, determinando aumento da tensão da parede, maior gasto energético e vazamento pelo aparelho valvar mitral.

Durante anos se considerou a proposta da correção da insuficiência mitral secundária, contraindicada para estas situações uma vez que o procedimento cirúrgico aumentava subitamente a póscarga após a eliminação da fração regurgitante.

Estudos dos últimos anos têm demonstrado que os pacientes melhoram após o procedimento com regressão de 1 ou mais classes funcionais (13-16).

Concebemos a idéia de corrigir a insuficiência mitral através do implante de uma prótese valvular em posição mitral e promover a remodelação ventricular esquerda com a técnica descrita neste trabalho.

Apresentamos aqui os resultados obtidos com a aplicação deste conceito em 33 pacientes consecutivamente operados de dezembro de 1995 a setembro de 1999.

\section{CASUÍSTICA E MÉTODOS}

Foram analisados 33 pacientes com insuficiência cardíaca refratária à terapêutica clínica máxima e que apresentavam insuficiência mitral moderada ou grave pela ecocardiografia transesofágica, por ocasião da indicação operatória. Todos os pacientes haviam tido pelo menos 3 internações hospitalares nos últimos 6 meses.

As idades variáveis de 25 a 78 anos (mediana 58 anos) e os períodos de seguimento pós-operatório variaram de 4 meses a 4 anos (mediana 22 meses). Quanto ao sexo, 19 pacientes eram do masculino e 14 do feminino.

No que diz respeito à etiologia da cardiomiopatia em fase terminal, em 15 pacientes era isquêmica, em 13 cardiomiopatia dilatada sem etiologia indicável, em 3 era chagásica, em 1 pós-parto e em 1 viral.

As classes funcionais pós-tratamento médico otimizado por ocasião da indicação operatória eram III (9 casos) e IV (24 casos) da NYHA. Sendo que 7 pacientes foram submetidos a operação dependentes de balão intra-aórtico.

Oito $(24,2 \%)$ pacientes haviam sido submetidos a operação cardíaca prévia e todos foram recusados para transplante cardíaco devido a contra-indicações de ordem clínica ou locais. 
Buffolo E, Paula I M, Branco J N R, Carvalho A C C, Mantovani C, Caputi G, Aguiar L F - Tratamento da insuficiência cardíaca terminal através da correção da insuficiência mitral secundária e remodelação ventricular. Rev Bras Cir Cardiovasc 2001; 16(3): 203-11.

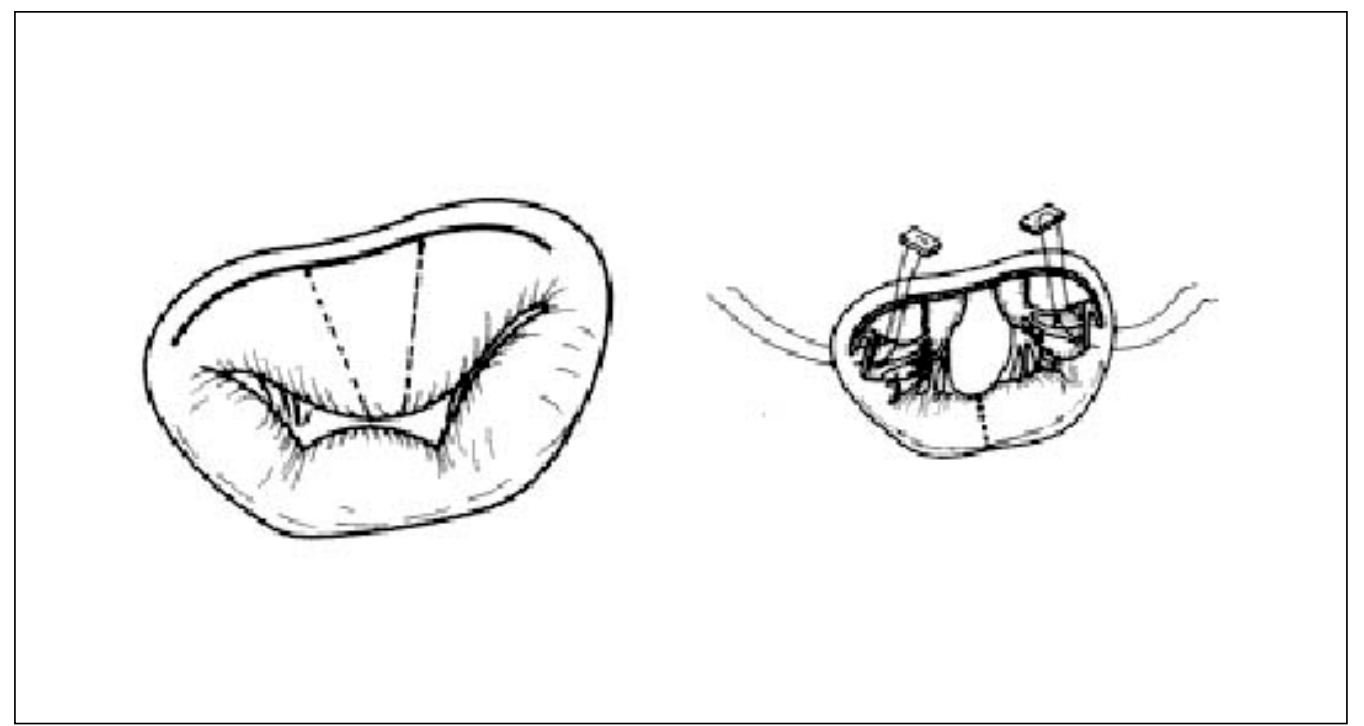

Fig 1 - Desenho esquemático da técnica da abertura da "janela" no folheto anterior e o local de aplicação dos pontos no vértice dos triângulos levando-os às comissuras. A fixação dos pontos ao teflon da prótese valvular, assim como, o pregueamento das lacunas ao anel traciona a ponta dos papilares para cima.

\section{Seleção de Pacientes}

Todos os pacientes, com exceção de um, apresentavam insuficiência mitral moderada ou severa à ecocardiografia transesofágica.

Não se indicou o procedimento para pacientes sem regurgitação mitral nas avaliações seriadas, mas se considerou para indicação cirúrgica pacientes que na evolução apresentavam insuficiência mitral indiscutível nos períodos de descompensação.

A ecocardiografia transtorácica convencional assim como a ventriculografia esquerda não foram valorizadas em demasia, em virtude de não evidenciarem insuficiência mitral expressiva mesmo em baixo desempenho do ventrículo esquerdo.

Em 7 dos 33 pacientes analisados, o estudo da função e da forma do ventrículo esquerdo através da ressonância nuclear magnética mostrou-se particularmente útil, em especial para comparação com os resultados pós-cirúrgicos.

Em $8(24,2 \%)$ casos foram realizados procedimentos associados: revascularização do miocárdio em 6 pacientes e exclusão de áreas acinéticas e revascularização em 2 , mas em nenhum deles o procedimento associado teria sido indicado isoladamente pela desprezível importância e impacto que teria na evolução natural da miocardiopatia.

Todos os pacientes foram estudados através de avaliações clínicas convencionais, eletrocardiografia, ecocardiografia transtorácica seriada, ecodopplercardiografia transesofágica, estudo hemodinâmico e cinecoronariografia. Sete pacientes foram submetidos a estudo da forma ventricular e refluxo mitral pela ressonância nuclear magnética.

\section{Técnica Operatória}

Todos os pacientes foram operados através de esternotomia mediana longitudinal, circulação extracorpórea com canulação de aorta e cavoatrial.

A valva mitral foi abordada em todos os casos por atriotomia esquerda e não apresentava lesão orgânica dos folhetos, sendo a insuficiência mitral devida a distorção dos músculos papilares pela dilatação ventricular.

O implante da prótese preservou os folhetos anteriores e posteriores sendo que o folheto posterior foi pregueado no anel posterior e o anterior seccionado em sua posição mediana, sendo os 2 vértices levados às comissuras correspondentes, abrindo-se uma janela para a via de saída do ventrículo esquerdo (Figura 1).

Estas manobras preservam a função dos músculos papilares e enxertam o eixo longitudinal do coração na distância correspondente que vai da borda livre dos folhetos até o anel atrioventricular esquerdo. Técnica semelhante foi descrita anteriormente por MIKI et al. (22) .

Em todos os pacientes utilizamos próteses de pequenos diâmetros, considerando o diâmetro do anel: em 18 pacientes foram empregadas próteses de $29 \mathrm{~mm}$, em 12 casos foram usadas próteses de 
Buffolo E, Paula I M, Branco J N R, Carvalho A C C, Mantovani C, Caputi G, Aguiar L F - Tratamento da insuficiência cardíaca terminal através da correção da insuficiência mitral secundária e remodelação ventricular. Rev Bras Cir Cardiovasc 2001; 16(3): 203-11.

$31 \mathrm{~mm}$, em 2 pacientes as próteses tinham $33 \mathrm{~mm}$. Em 1 caso a prótese foi implantada com a finalidade de remodelar a base do ventrículo esquerdo. Em 31 casos foram utilizadas próteses biológicas e em 2 mecânicas.

A cardioplegia utilizada foi a sangüínea anterógrada intermitente a $28^{\circ} \mathrm{C}$ e em 6 pacientes o implante da prótese foi realizado sem pinçamento aórtico.

As operações foram realizadas após aprovação dos pacientes e das Instituições.

\section{Seguimento Pós-Operatório}

Todos os pacientes foram vistos por 2 médicos sendo as indicações operatórias decididas em consenso.

A hipótese central assumida era que poderia haver melhora da classe funcional, sendo as avaliações feitas em 3 etapas: imediatamente antes da operação, antes da alta hospitalar e na última e mais recente avaliação do paciente.

Além das avaliações clínicas, foram realizados estudos ecocardiográficos seriados para análise de dois parâmetros básicos: fração de ejeção e volume efetivo do VE.

Para estas análises utilizamos teste de variâncias de Friedman, sendo o erro estabelecido em $p<0,01$.

\section{RESULTADOS}

Dos 33 pacientes operados, observamos 4 $(12,1 \%)$ óbitos na fase hospitalar devido a falência ventricular esquerda e baixo débito cardíaco (2 casos), falência de múltiplos órgãos (1) e acidente vascular cerebral no $3^{\circ}$ dia pós-operatório (1 caso).

Dos 29 pacientes que receberam alta hospitalar e foram acompanhados ambulatorialmente, 3 faleceram: aos 2 meses de evolução (fibrilação ventricular), aos 6 meses (insuficiência cardíaca) e aos 23 meses (hepatite, insuficiência hepática).

Três pacientes foram reoperados por vazamento perivalvar sem óbitos, recuperando a melhora por vazamento perivalvar sem óbitos e a melhoria perdida da classe funcional.

Estes foram pacientes da fase inicial, na qual não se ancorava os pontos em teflon. Após esta tática não tivemos mais casos de deiscência de sutura da prótese.

No período de pós-operatório imediato a maioria dos pacientes necessitou do apoio de inotrópicos por alguns dias, sendo o desmame farmacológico e do balão intra-aórtico realizado progressivamente. A curva atuarial de sobrevivência pode ser observada no Gráfico 1.

Após a operação dos 27 sobreviventes com mais de 6 meses de observação, 23 estão em classe funcional II e 4 em classe funcional III com apoio medicamentoso. A análise de variância com testes de Friedmam revela que a melhora é significativamente importante $(p<0,0001)$ e se manteve ao longo do período (Gráfico 2).

A análise seriada da fração de ejeção do ventrículo esquerdo aferida através da ecocardiografia transtorácica convencional nos períodos: pré-operatório, antes da alta hospitalar e na última evolução do paciente com um período mínimo de seguimento de 6 meses demonstrou melhora não expressiva variando de 30 para 32 e 36 (Gráfico 3).

Em compensação, o volume ejetivo do ventrículo esquerdo aferido nas 3 fases demonstrou aumento significativo variando de $50 \mathrm{ml}$ no pré-operatório, 69 $\mathrm{ml}$ antes da alta hospitalar e $80 \mathrm{ml}$ na última evolução do paciente (Gráfico 4).

Os diâmetros diastólico e sistólico do ventrículo esquerdo não variaram significativamente, mas nos 7 casos em que a geometria ventricular pode ser estudada através da ressonância nuclear magnética observou-se uma remodelação da base do coração esquerdo e da região septal.

\section{COMENTÁRIOS}

Táticas cirúrgicas alternativas utilizadas no tratamento da insuficiência cardíaca refratária não atingiram as expectativas de grande impacto no curso natural da afecção. Porcentual expressivo de pacientes não mostraram maior ou melhor qualidade de vida nos seguimentos pós-operatórios.

Estas operações demonstraram alta mortalidade (ventriculectomia parcial), degeneração do enxerto muscular (cardiomioplastia) ou resolução incompleta da insuficiência cardíaca para as demais proposições.

A concepção de corrigir a insuficiência mitral através de plastia ou substituição valvar proposta por BOLLING et al. (15) demonstrou ser este procedimento válido e de resultados confiáveis $(13,14,16)$.

A técnica descrita neste trabalho propõe o implante de uma bioprótese de tamanho reduzido no anel atrioventricular esquerdo, com a finalidade de remodelar a base ventricular (musculatura circular) e encurtar o eixo longitudinal do ventrículo esquerdo pela tração da ponta dos músculos papilares até próximo 
Buffolo E, Paula I M, Branco J N R, Carvalho A C C, Mantovani C, Caputi G, Aguiar L F - Tratamento da insuficiência cardíaca terminal através da correção da insuficiência mitral secundária e remodelação ventricular. Rev Bras Cir Cardiovasc 2001; 16(3): 203-11.

\section{GRÁFICO 1}

CURVA ATUARIAL DE SOBREVIVÊNCIA DOS 33 PACIENTES OPERADOS, PORTADORES DE CARDIOMIOPATIA DILATADA GRAVE E CIRURGIA VALVAR MITRAL

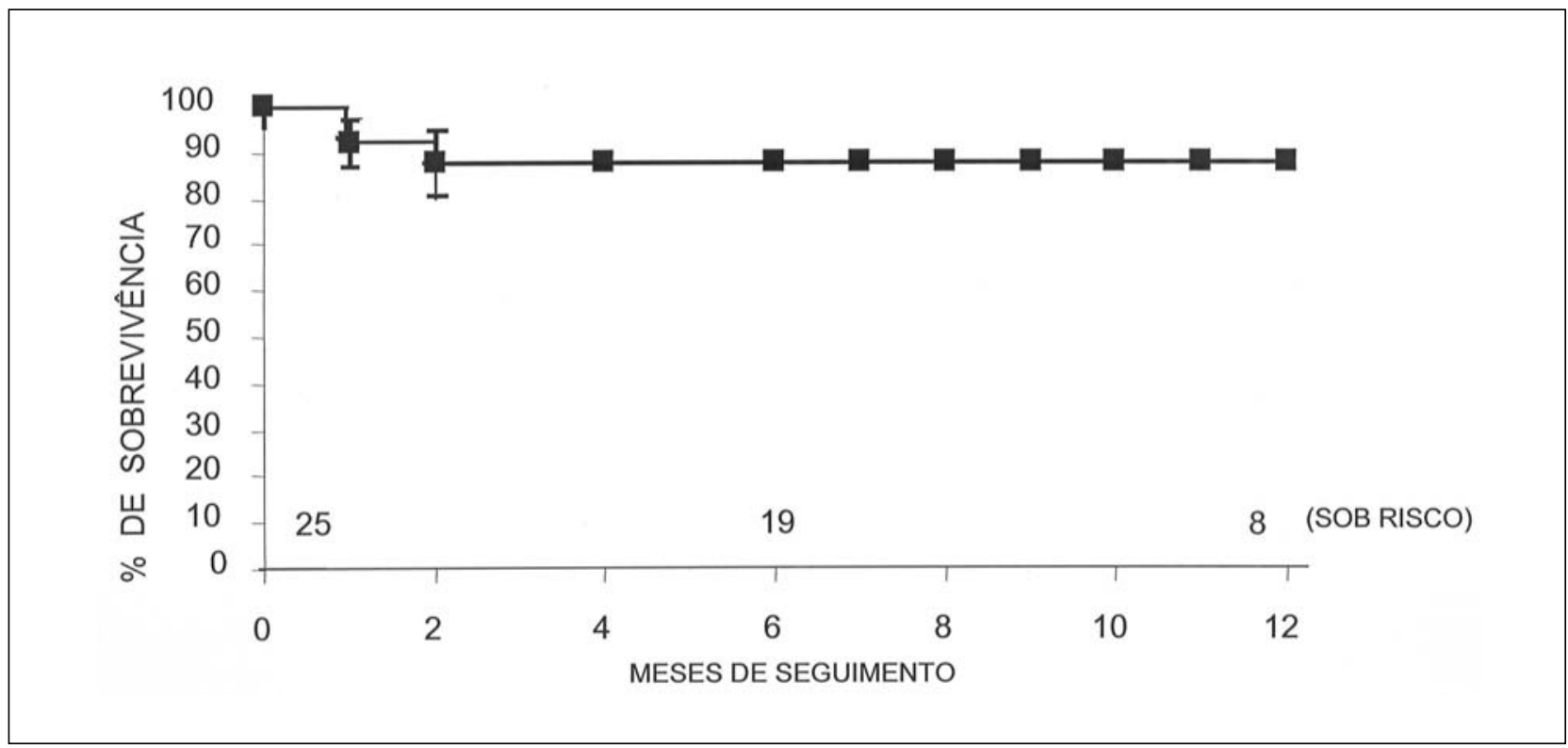

GRÁFICO 2

CLASSES FUNCIONAIS PRÉ E PÓS-OPERATÓRIAS

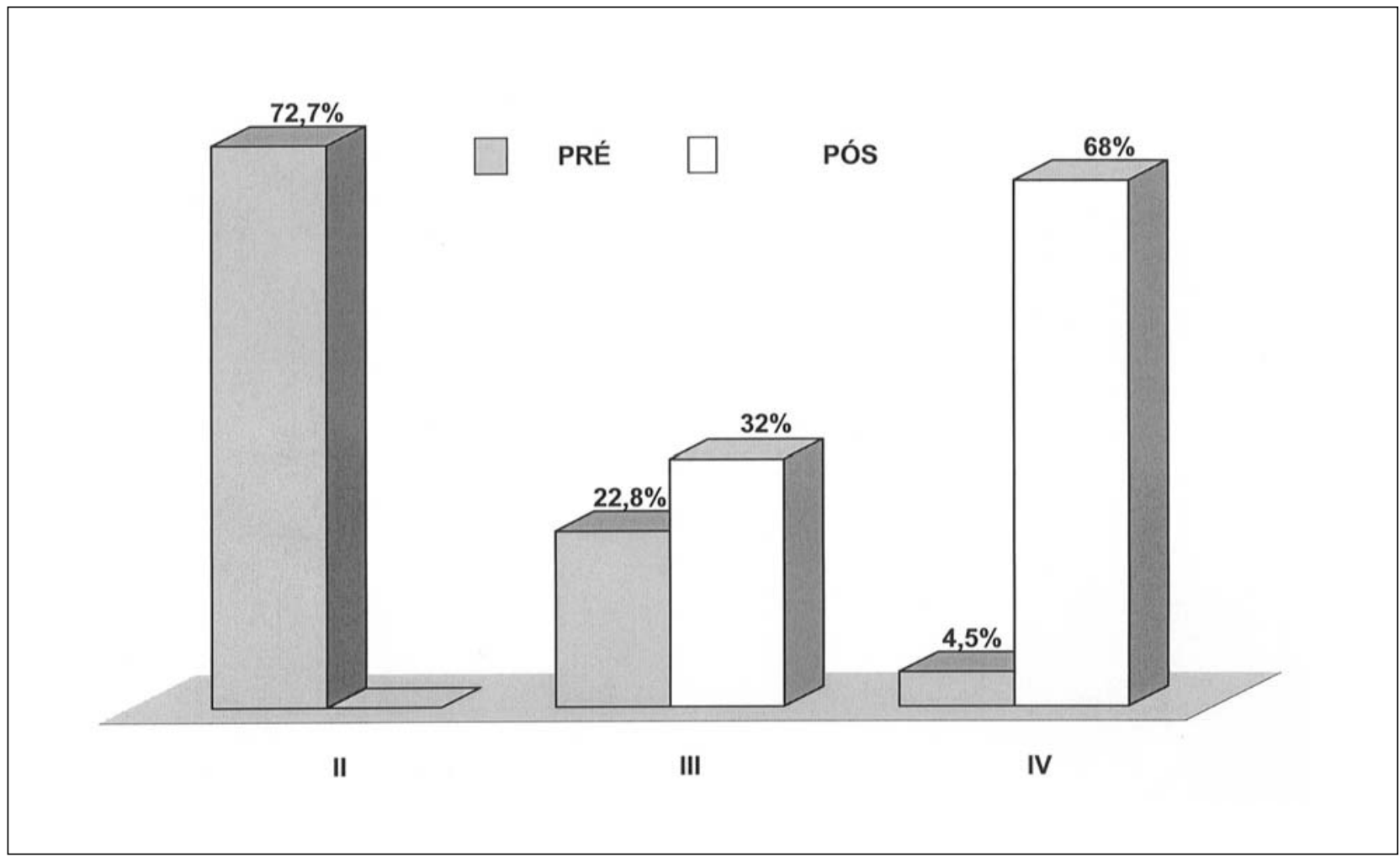


Buffolo E, Paula I M, Branco J N R, Carvalho A C C, Mantovani C, Caputi G, Aguiar L F - Tratamento da insuficiência cardíaca terminal através da correção da insuficiência mitral secundária e remodelação ventricular. Rev Bras Cir Cardiovasc 2001; 16(3): 203-11.

\section{GRÁFICO 3}

EVOLUÇÃO DA FRAÇÃO DE EJEÇÃO ECOCARDIOGRÁFICA DO VENTRÍCULO ESQUERDO NAS FASES PRÉ-OPERATÓRIA IMEDIATA, ANTES DA ALTA HOSPITALAR E NA ÚLTIMA (MAIS RECENTE) AVALIAÇÃO

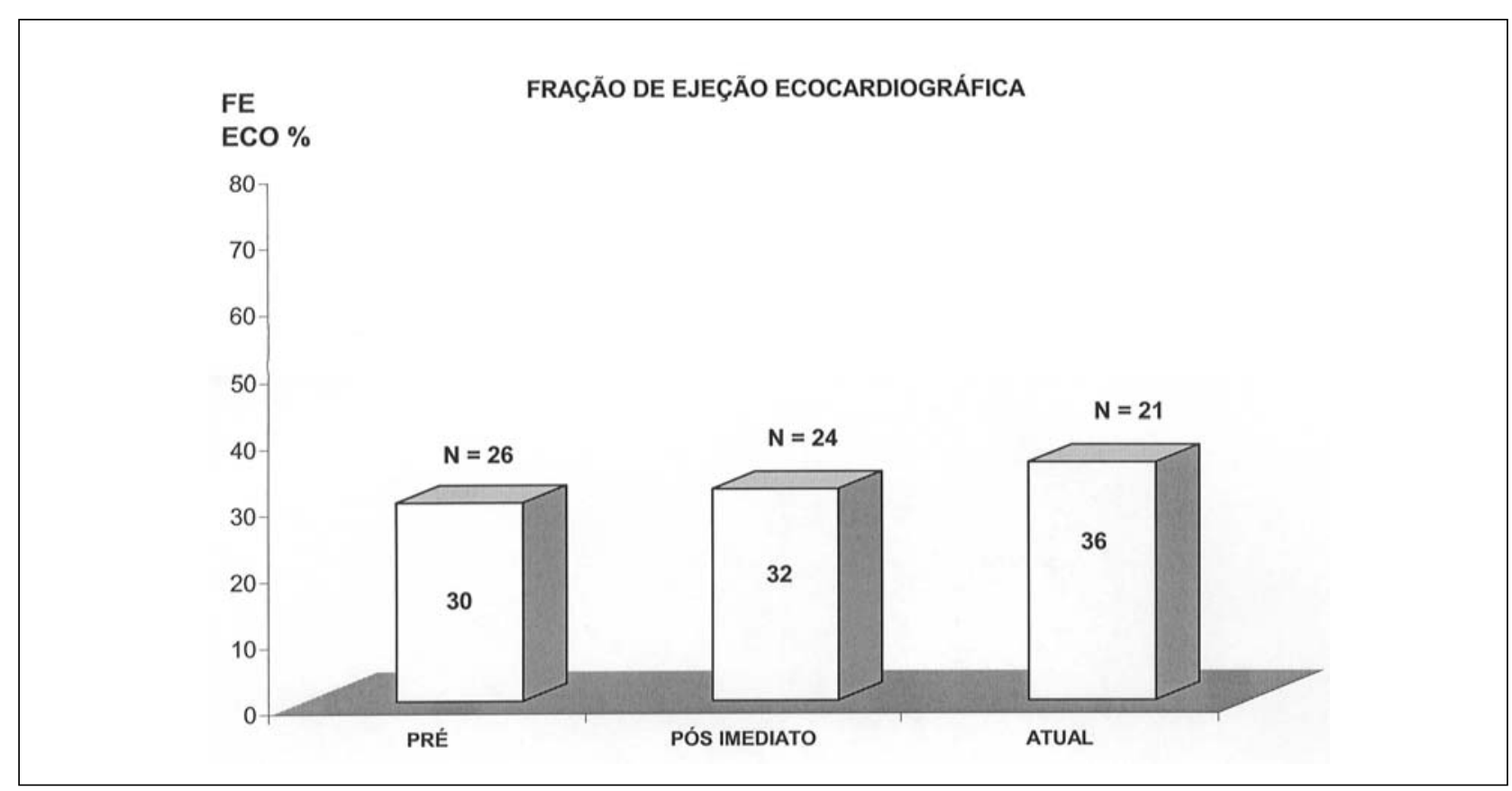

GRÁFICO 4

EVOLUÇÃO DO VOLUME EFETIVO DO VENTRÍCULO ESQUERDO, OBSERVANDO-SE MELHORIA CONSIDERÁVEL DO VOLUME E DISCRETA MELHORA DA FRAÇÃO DE EJEÇÃO

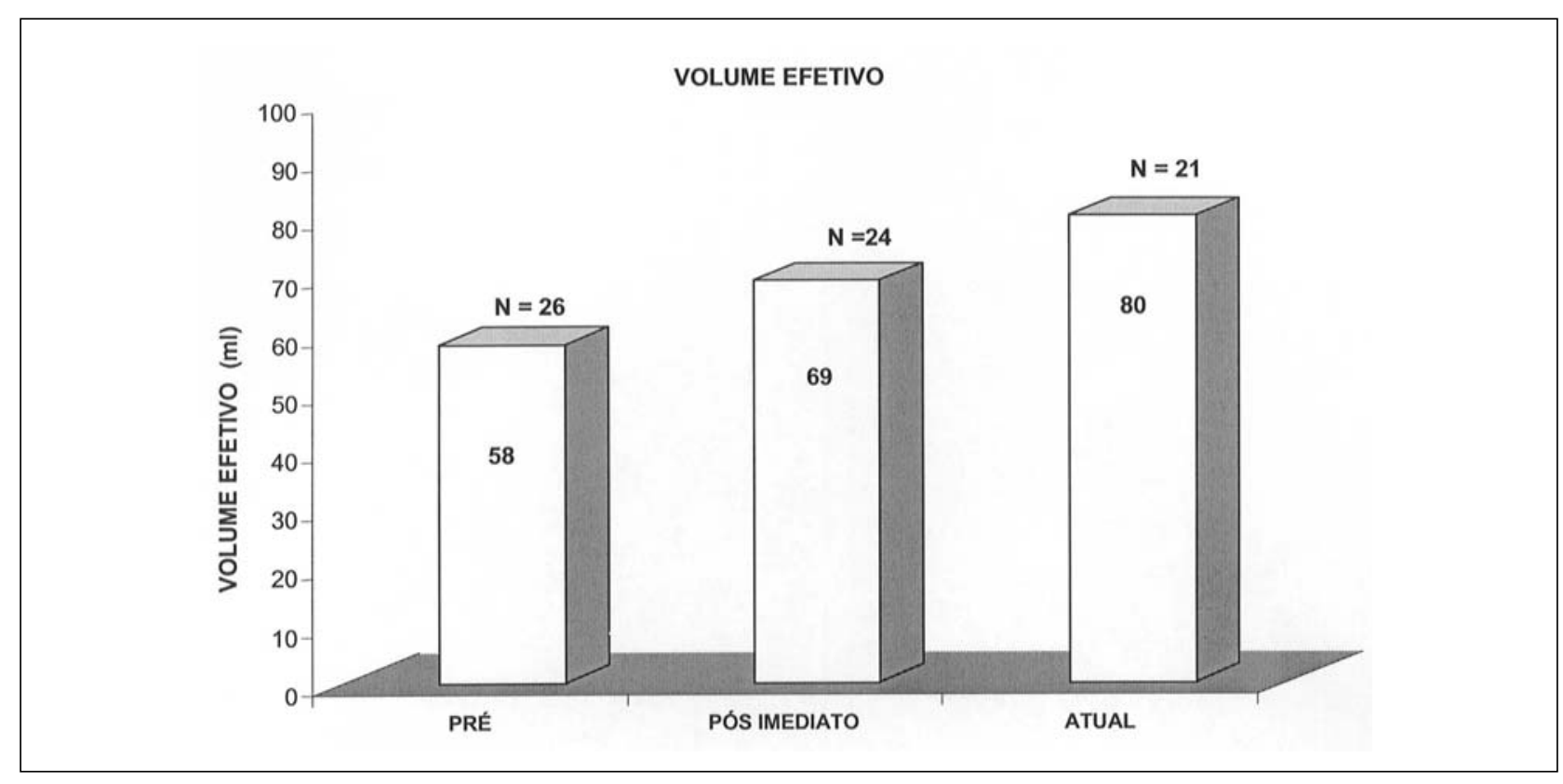


Buffolo E, Paula I M, Branco J N R, Carvalho A C C, Mantovani C, Caputi G, Aguiar L F - Tratamento da insuficiência cardíaca terminal através da correção da insuficiência mitral secundária e remodelação ventricular. Rev Bras Cir Cardiovasc 2001; 16(3): 203-11.

ao anel atrioventricular (musculatura sino-espiral) com o pregueamento da borda das cúspides do anel. Com a técnica descrita consegue-se a eliminação da regurgitação de uma maneira simples e eficiente, a remodelação do ventrículo esquerdo e a conservação da função papilar que é indiscutivelmente eficiente ${ }^{(23)}$.

O inconveniente do implante de uma prótese valvular é a nosso ver irrelevante, pois provavelmente a expectativa de vida do paciente é menor que a vida da bioprótese e se isto não ocorresse seria a confirmação de um sucesso extraordinário.

A correção da insuficiência mitral e a remodelação ventricular proposta neste trabalho demonstraram melhoria significativa da classe funcional pré-operatória de pacientes terminais. Esta melhora clínica teve apoio objetivo através da melhora do volume ejetivo do ventrículo esquerdo, apesar da melhora descrita da fração de ejeção. Isto significa que a eliminação da fração regurgitante foi a responsável por esta melhoria.

São necessários estudos futuros para analisar melhor a modificação da geometria ventricular obtida com esta técnica operatória e, para tanto, estudos prospectivos com aplicação de ressonância nuclear estão sendo realizados.
Uma limitação deste estudo diz respeito ao fato de 8 dos 33 pacientes analisados terem sido submetidos a procedimentos associados, todavia em nossa opinião foram de pouco impacto, uma vez que isoladamente jamais teriam a indicação operatória realizada.

Apesar do seguimento médio (22 meses) ser relativamente curto, esta experiência demonstrou que pode ser oferecida a pacientes com miocardiopatia e insuficiência cardíaca refratária, constituindo uma alternativa ao transplante cardíaco desde que seja documentada insuficiência mitral pelo menos moderada.

\section{CONCLUSÃO}

A técnica apresentada neste trabalho de correção da insuficiência mitral secundária em pacientes com insuficiência cardíaca refratária constitui-se em promissora operação alternativa ao transplante cardíaco com aceitável risco operatório e melhoria da classe funcional.

RBCCV 44205-548

Buffolo E, Paula I M, Branco J N R, Carvalho A C C, Mantovani C, Caputi G, Aguiar L F - Treatment of terminal cardic insufficiency by means of correction of secondary insufficiency and ventricular remodelling. Rev Bras Cir Cardiovasc 2001; 16(3): 203-11.

ABSTRACT: Introduction: The survival of patients in end-stage and secondary mitral insufficiency is very poor in short periods of follow-up in spite of the progress that has been made in clinical management. The occurrence of secundary mitral regurgitation compromises survival and quality of life and recent papers have suggested that mitral intervention could improve functional classes.

Objective: This paper describes a techniques of valvular prostheses implantation in the left A-V annulus, to correct mitral regurgitation and remodelling the basis of the left ventricle with shortening of the longitudinal axis.

Material and Methods: We analyze 33 patients in end-stage cardiomyopathies (15 ischemic, 13 dilated, 3 Chagas' disease, 1 puerperal, 1 viral) operated on from December 85 to September 99. The ages varied from 25 to 78 years (mean 58), 57.7\% males. The period of follow-up was from 4 months until 4 years (mean 22 months).

Results: During hospital stay, 3 deaths (3/33) occurred and 2 others occurred after hospital discharge $(2 / 30)$. In this period of follow-up $88 \%$ of patients improved 1 or 2 functional classes, the echocardiographic ejection fraction improved from 30 to $36 \%$ despite elimination of regurgitant flow and the stroke volume increased from 58 to $80 \mathrm{ml}$.

Conclusion: Refractory cardiac insufficiency with mitral regurgitation can be palliative with valvular prosthesis implantation and remodelling of left ventricle in this period of follow-up.

DESCRIPTORS: Cardiac output, low, surgery. Mitral valve insufficiency, surgery. Ventricular remodeling. Heart valve prosthesis implatation. Cardiac output, low, complications. Mitral valve insufficiency, etiology. Cardiac output, low, survival analysis. 
Buffolo E, Paula I M, Branco J N R, Carvalho A C C, Mantovani C, Caputi G, Aguiar L F - Tratamento da insuficiência cardíaca terminal através da correção da insuficiência mitral secundária e remodelação ventricular. Rev Bras Cir Cardiovasc 2001; 16(3): 203-11.

\section{REFERÊNCIAS BIBLIOGRÁFICAS}

1 Evans R W, Orians C E, Ascher N L - The potential supply of organ donors: an assessment of the efficacy of organ procurement efforts in the United States. JAMA 1992; 267:239-46.

2 Bassie B M \& Parker M. - Congestive heart failure: current controversies in future projects. $A m \mathrm{~J}$ Cardiol 1990; 66: 429-30.

3 Bourassa M G, Gurne O, Bangdiwala S I et al. - Natural history and patterns of current practice in heart failure: the studies of left ventricular dysfunction investigators. J Am Coll Cardiol 1993; 22 (4 suppl A): $14 \mathrm{~A}-9$.

4 Carpentier A, Chachques J C, Acar A et al.- Dynamic cardiomyoplasty at seven years. J Thorac Cardiovasc Surg 1993; 106: 42-54.

5 Patel H J, Lankford E B, Polidori D J et al. - Dynamic cardiomyoplasty: its chronic and acute effects on the failing heart. J Thorac Cardiovasc Surg 1997; 114:169-78.

6

El Oakley R M \& Jarvis J C - Cardiomyoplasty: a critical review of experimental and clinical results. Circulation 1994; 90:2085-90.

7 Moreira L F, Stolf N A, Braile D M, Jatene A D Dynamic cardiomyoplasty in South America. Ann Thorac Surg 1996; 61: 408-12.

8 Batista R J, Santos J L, Takeshita N, Bocchino L, Lima P N, Cunha M A - Partial left ventriculectomy to improve left ventricular function in end-stage heart disease. J Card Surg 1996; 11: 96-8.

9 McCarthy P M, Starling R C, Wong J et al. - Early results with partial left ventriculectomy. J Thorac Cardiovasc Surg 1997; 114:755-65.

10 Batista R J, Verde J, Nery P et al. - Partial left ventriculectomy to treat end-stage heart disease. Ann Thorac Surg 1997; 64:634-8.

11 Repogle R L \& Kaiser G C - Position paper: new techonology asserment commitee of left ventricular reduction. Ann Thorac Surg 1997; 63:909-10.

12 Dor V, Sabatier M, Donato M, Montiglio F, Toso A, Maioli M - Efficacy of endoventricular patch plasty in large postinfarction akinetic scar and severe left ventricular dysfunction: comparison with a series of large dyskinetic scars. J Thorac Cardiovasc Surg 1998; 116:50-9.

13 Bolling S F, Pagani F D, Deeb G M, Bach D S Intermediate-term outcome of mitral reconstruction in cardiomyopathy. J Thorac Cardiovasc Surg 1998; 115:381-8.

14 Bach D S \& Bolling S F - Early improvement in congestive heart failure after correction of secondary mitral regurgitation. Am Heart $J$ 1995; 129:1165-70.

15 Bolling S F, Deeb G M, Brunsting L A, Bach D S Early outcome of mitral valve reconstruction in patients with end-stage cardiomyopasthy. $J$ Thorac Cardiovasc Surg 1995; 1099:676-83.

16 Calafiore A M, Gallina S, Contini M et al. - Surgical treatment of dilated cardiomyopathy with conventional techniques. Eur J Cardiothorac Surg 1999; 16(suppl 1):73-8.

17 Wilensky R L, Yudelman P, Cohen A I et al. - Serial electrocardiographic changes in idiopathic dilated cardiomyopathy confirmed at necropsy. $A m \mathrm{~J}$ Cardiol 1998; 62:276-83.

18 Xiao H B, Lee C H, Gibson D G - Effect of left bundle branch block on diastolic function in dilated cardiomyopathy. Br Heart J 1991; 66:443-7.

19 Alonso C, Leclercq C, Victor F et al. - Electrocardiographic predictive factors of long-term clinical improvement with multisite biventricular pacing in advanced heart failure. Am J Cardiol 1999; 84:1417-21.

20 Blanc $\mathrm{J} J$, Etienne $\mathrm{Y}$, Gilard $\mathrm{M}$ et al. - Evaluation of different ventricular pacing sites in patients with severe heart failure: results of an acute hemodynamic study. Circulation 1997; 96:3273-7.

21 Boltwood C M, Tei C; Wong M, Shah P M - Quantitative echocardiography of the mitral complex in dilated cardiomyopathy: the mechanism of functional mitral regurgitation. Circulation 1983; 68:498-508.

22 Miki S, Kusuhara K, Ueda Y, Komeda M, Ohkita Y, Tahata $T$ - Mitral valve replacement with preservation of chordae tendineae and papillary muscles. Ann Thorac Surg 1988; 45:28-34.

23 Lillehei C W, Levy M J, Bonnabeau R C - Mitral valve replacement with preservation of pappilary muscles and chordae tendineae. J Thorac Cardiovasc Surg $1964 ; 47: 532-43$. 
Buffolo E, Paula I M, Branco J N R, Carvalho A C C, Mantovani C, Caputi G, Aguiar L F - Tratamento da insuficiência cardíaca terminal através da correção da insuficiência mitral secundária e remodelação ventricular. Rev Bras Cir Cardiovasc 2001; 16(3): 203-11.

\section{Discussão (Transcrições de fita gravada)}

\author{
DR. SÉRGIO ALMEIDA DE OLIVEIRA \\ São Paulo, SP
}

Agradeço aos membros da Comissão Organizadora pelo convite para comentar este trabalho. O tema é de grande atualidade e vem sendo uma preocupação de todos nós que tratamos pacientes com insuficiência cardíaca terminal. As alternativas para o transplante cardíaco são várias e muitas delas podem ser utilizadas em associação. A insuficiência mitral na cardiomiopatia dilatada, não isquêmica e sem lesão primária da valva mitral, é predominantemente conseqüência da dilatação anular progressiva, com perda da coaptação das cúspides. Na cardiomiopatia dilatada isquêmica, o mecanismo que leva à insuficiência mitral é mais complexo e, além da insuficiência funcional pela dilatação anular, inclue também todo o aparelho mitral e a parede do ventrículo esquerdo (VE). A sobrecarga de volume, secundária à insuficiência mitral, leva à dilatação ventricular e disfunção, a qual acarreta mais dilatação anular. A correção da insuficiência mitral pode interromper este ciclo vicioso, ajudando na compensação. Um outro mecanismo pelo qual a reconstrução da valva mitral ajuda este coração dilatado é o da remodelação da base do ventrículo, pela redução do anel, restabelecendo a forma elipsóide da base do ventrículo. A área da valva mitral normal é muito grande e permite que possamos reduzíla consideravelmente. Gostaria de perguntar por que não dar preferência para a plástica mitral, com redução do hemi-anel posterior, e realinhamento dos músculos papilares? Em seus 33 pacientes, próteses $n^{\circ} 27$ foram usadas em 18 deles e próteses 29, 31 ou 33 usadas em 15 pacientes. Não seriam estes últimos números muito grandes para esta finalidade? Quando tenho que trocar a valva nesta situação prefiro usar a manobra descrita por Willian Angell. A cúspide anterior é desinserida e fixada à cúspide posterior, conservando todo o aparelho valvar. Nos casos de miocardiopatia isquêmica, gostaria de lembrar que, além da cirurgia sobre a valva mitral, e da revascularização da área isquêmica, tem grande importância a identificação de áreas acinéticas ântero-septal, que podem ser corrigidas pela operação de Jatene ou de Dor. Tem sido dada muita atenção às áreas discinéticas ou aneurismáticas, mas ultimamente temos atribuído às áreas acinéticas ântero-septal a mesma importância. O que você acha deste problema? Parabenizo o Dr. Enio e sua equipe por esta linda apresentação, especialmente pelo rigoroso seguimento pósoperatório.

\section{DR. BUFFOLO}

(Encerrando)

Gostaria de agradecer aos comentários e no que diz respeito à indagação sobre a substituição valvar, eu propositadamente faço a substituição valvar porque acho que se tivermos problema com a prótese 7-8 anos depois, o resultado seria excelente. O implante da prótese é uma operação de segurança neste tipo de paciente, é rápida, confiável e todos nós somos capazes de fazer em 30-35 minutos, em alguns casos com o coração batendo. Além disso, colocar uma prótese permite uma remodelação segura da base do coração. Vocês podem dizer que o coração poderia ser remodelado com plastias habituais, mas não conseguiríamos fazer a tração dos músculos papilares. Não estou satisfeito ainda com a maneira como a gente está fazendo a suspensão dos músculos papilares, tenho certeza que cada um de nós vai pensar e vai descobrir uma maneira mais inteligente de fazer isso. Tenho conversado como Dr. Braile que retira um segmento oval da cúspide anterior e consegue atingir o objetivo. A possibilidade de cruzar os músculos papilares também é atraente, só que não posso ficar tentando uma técnica sobre a outra pois não vou chegar a lugar algum; é preciso insistir em uma linha para poder tirar as conclusões. Outra coisa que eu aprendi com esta técnica é que o septo tem um componente muito importante. Acho que o princípio do Randas é perfeito e nós temos que perseguir isto, se houvesse uma forma de fazer a operação as custas do septo, tenho a impressão de que seria formidável. Quanto ao último comentário do Dr. Sérgio sobre as áreas acinéticas do ventrículo esquerdo, estes doentes não estão nesta casuística, não é destes doentes que estou falando. Tenho insistido na necessidade do diagnóstico difícil da discinesia no indivíduo com baixa fração de ejeção. O indivíduo que tem um aneurisma, na fase final, ocorre dilatação da porção basal e o aneurisma não é diagnosticado, aparecendo na operação ou ainda descobrimos uma área acinética na parede anterior. Reconheço que existem vários procedimentos associados, os quais só não retirei deste material porque, na minha opinião, não estou cometendo nenhum pecado mortal. O que chamamos de ressecção do aneurisma neste pacientes é uma plicatura de pequena área da parede anterior e nunca seria este o motivo da operação. Tive alguma dificuldade em selecionar os doentes que seriam incluídos neste casuística, excluí a maioria e deixei apenas aqueles realmente graves. 